\title{
Evaluation of the effect of apixaban using a viscoelastic coagulation assay with Russell's viper venom reagent
}

\author{
Kaoru Suzuki ${ }^{1}$, Nobuyuki Katori ${ }^{1 *}$ (D, Y Yoshihiro Kimura ${ }^{1}$, Takako Terui ${ }^{1}$, Hiroshi Sunaga', Shunsuke Kobayashi ${ }^{2}$ and \\ Shoichi Uezono ${ }^{1}$
}

\begin{abstract}
Background: Conventional coagulation tests, such as prothrombin time and activated partial thromboplastin time, are not sensitive to anticoagulation by apixaban. We evaluated the antithrombotic effect of apixaban using a Russell viper venom (RW) test for a patient who underwent posterior spine fusion surgery.

Case presentation: An 84-year-old man was scheduled for percutaneous posterior spine fusion. He continued apixaban until the night before surgery and resumed it on the first day after surgery. We performed an RW test as point-of-care coagulation monitoring in combination with chromogenic anti-activated factor X (anti-Xa) activity, prothrombin time, and activated partial thromboplastin time. Clotting time with the RW test was prolonged according to the anti-Xa activity of apixaban, which was in the therapeutic range during surgery.

Conclusions: An RW test might be useful as a point-of-care assay for estimation of the anti-Xa level induced by apixaban during the perioperative period.
\end{abstract}

Keywords: Anticoagulation, Anti-Xa, Apixaban, Russell's viper venom

\section{Background}

Conventional coagulation tests, such as prothrombin time (PT) and activated partial thromboplastin time (APTT), for monitoring of the anticoagulant effect of direct oral anticoagulants are not always required. However, there are circumstances, including emergency surgery, trauma, life-threating bleeding, acute kidney injury, etc., in which assessment of anticoagulant effect would be desirable. Although PT and APTT are the conventional coagulation tests used for the monitoring of anticoagulant drugs, such as warfarin and heparin, these tests are not sensitive to direct oral anti-activated factor $\mathrm{X}$ (anti-Xa) drugs such as apixaban [1].

Recently, a point-of-care, whole-blood, and viscoelastic device that uses active chip technology (ClotPro; enicor

\footnotetext{
* Correspondence: nbykktr@gmail.com

'Department of Anesthesiology, The Jikei University School of Medicine,

3-25-8 Nishishinbashi, Minato-ku, Tokyo 105-8461, Japan

Full list of author information is available at the end of the article
}

$\mathrm{GmbH}$, Munich, Germany) has been approved for clinical use. In addition to established thromboelastometry assays, ClotPro provides a unique assay that can evaluate the inhibitory effect on Xa using Russell's viper venom (RVV), which has high sensitivity to anti-Xa drugs. We evaluated the antithrombotic effect of apixaban using ClotPro and RRV for a patient who underwent posterior spine fusion surgery.

\section{Case presentation}

The patient was an 84-year-old man with a height of $176 \mathrm{~cm}$ and a body weight of $80 \mathrm{~kg}$ who was scheduled for minimally invasive posterior spine fusion (T9-L3) for the treatment of a lumbar compression fracture. $\mathrm{He}$ had a history of hypertension, atrial fibrillation, and chronic kidney disease and was taking amlodipine and apixaban (5 mg twice daily). Preoperative serum examination indicated moderate loss of kidney function, which was confirmed by a serum creatinine level of $1.05 \mathrm{mg} / \mathrm{dL}$ 
and an estimated glomerular filtration rate of $52 \mathrm{~mL} /$ $\mathrm{min} / 1.73 \mathrm{~m}^{2}$. Preoperative coagulation tests indicated prolonged values for both APTT and PT-international normalized ratio (40.1 s and 1.7, respectively). Although temporary interruption of apixaban is commonly recommended for the perioperative period (usually 48-72 $\mathrm{h}$ before surgical procedures, such as spine surgery, with high bleeding risk) [2], the patient continued apixaban until the night before surgery, according to the instruction of his cardiologist.

We considered it necessary to evaluate the residual anti-Xa effect of apixaban for the estimation of perioperative bleeding and potential need for transfusion; therefore, we implemented the use of ClotPro in combination with the conventional blood tests performed at our hospital's central laboratory. ClotPro uses RVV as the reagent to activate coagulation. Because RVV specifically cleaves factor $\mathrm{X}$ to $\mathrm{Xa}$, clotting time $(\mathrm{CT})$ is prolonged by anti-Xa drugs, such as apixaban, in a dosedependent manner [3, 4]. We examined blood cell count, PT, APTT, and plasma fibrinogen level at four time points: after anesthesia induction, at the end of surgery, on postoperative day (POD) 1, and on POD 6. We simultaneously performed the ClotPro assay, an ecarin chromogenic assay (ECA), to detect the effect of direct thrombin inhibitors such as dabigatran [5], and a fibrinogen concentration assay. Blood samples for outsourced chromogenic anti-Xa assay were collected at the same time points.

Anesthesia was induced with propofol and rocuronium, and was maintained with desflurane and continuous infusion of remifentanil. The results of coagulation tests after anesthesia induction indicated delayed thrombin production (Table 1) (Fig. 1). All members of the surgical team recognized the residual effects of apixaban, and we prepared fresh frozen plasma for excessive bleeding. Fortunately, the volume of bleeding was $440 \mathrm{~mL}$, and we did not perform a transfusion. Considering the balance of the risk of postoperative bleeding and the result of the RVV test on the morning of POD 1, which was in the normal range, we resumed apixaban at a decreased dose of $2.5 \mathrm{mg}$ twice daily on POD 1 . The CT for the RVV test on POD 6 was prolonged to $93 \mathrm{~s}$, which was shorter than the preoperative value (167 s) (Table 1). Although the patient had minor subconjunctival hemorrhaging after the resumption of apixaban, he was discharged without major bleeding or thrombotic complications.

\section{Discussion}

Major bleeding owing to the residual effect of apixaban was the major concern in the present case; however, the volume of blood loss was $440 \mathrm{~mL}$, and red blood cell transfusion was not required perioperatively. Although a percutaneous technique for posterior spine fusion might contribute to the moderate amount of bleeding, the perioperative bleeding risk for spine surgery is usually recognized as high [6]. The principles of perioperative management of antithrombotic therapy are interruption of antithrombotic drugs before surgery and prompt resumption after confirmation of surgical hemostasis. The European Heart Rhythm Association recommends $48 \mathrm{~h}$ or longer for the interruption period for direct oral anticoagulants before high-bleeding-risk interventions including spine surgery [7], although the timing of resumption depends on the balance between the risks of thrombosis and postprocedural bleeding in each patient. The present patient continued apixaban until the night before surgery; the duration between the last apixaban dose and the first blood sample collection in the operating room (after anesthesia induction) was $15 \mathrm{~h}$. Given the plasma terminal half-life of apixaban of approximately $10 \mathrm{~h}$ [8], we expected that the apixaban plasma concentration would be at approximately trough level at the start of surgery.

The values for PT and APTT after anesthesia induction were slightly prolonged, consistent with the increased plasma concentration of apixaban [9]. However, the relation between the plasma concentration of apixaban and PT or APTT is poor; the slopes of the

Table 1 Perioperative coagulation tests

\begin{tabular}{|c|c|c|c|c|c|}
\hline Coagulation test & Normal range & After anesthesia induction & At end of surgery & POD 1 & POD 6 \\
\hline Fibrinogen (mg/dL) & $150-400^{a}$ & 219 & 220 & 249 & 399 \\
\hline $\mathrm{APTT}(\mathrm{s})$ & $24-36^{\mathrm{a}}$ & 40.3 & 38.0 & 38.5 & 34.7 \\
\hline PT (\%) & $70-110^{a}$ & 42 & 49 & 59 & 69 \\
\hline PT-INR & $0.9-1.1^{\mathrm{a}}$ & 1.7 & 1.5 & 1.3 & 1.2 \\
\hline $\mathrm{CT}$ in RW test (s) & $48-77^{b}$ & 167 & 126 & 73 & 93 \\
\hline$C T$ in ECA test (s) & $68-100^{b}$ & 76 & 77 & 73 & 71 \\
\hline Anti-Xa activity (IU/mL) & $<0.1^{\mathrm{b}}$ & 1.69 & 1.12 & 0.06 & 0.78 \\
\hline
\end{tabular}

APTT Activated partial thromboplastin time, CT Clotting time, ECA Ecarin, INR International normalized ratio, POD Postoperative day, PT Prothrombin time, RVV Russell viper venom, $X a$ Activated factor $X$

a Based on the institutional reference values

${ }^{\mathrm{b}}$ Based on the manufacturer's reference values 


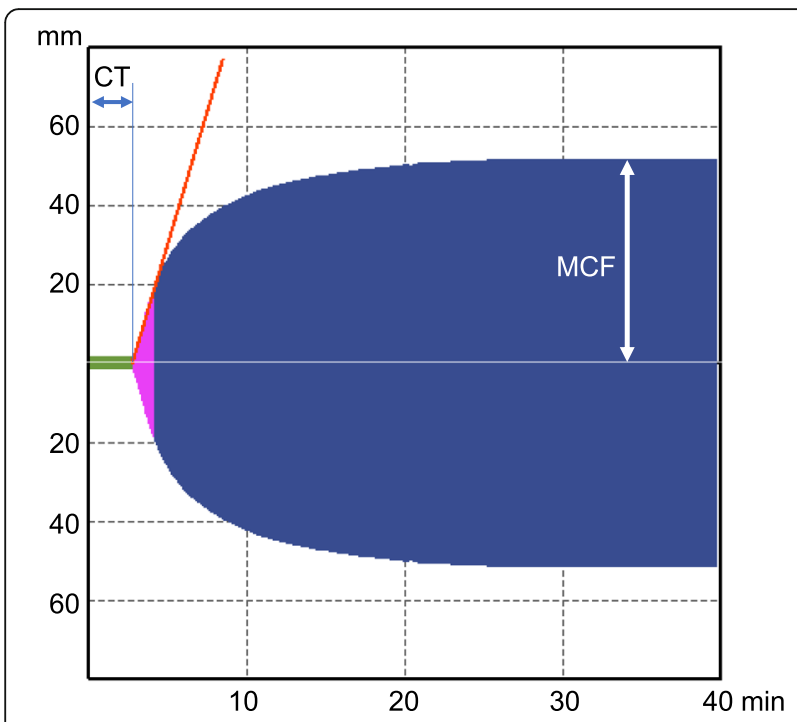

Fig. 1 A waveform of the RW-test after anesthesia induction. Clotting time $(\mathrm{CT})$ is prolonged to $167 \mathrm{~s}$ (normal range 48-77 s). CT is a time duration between initiation of the test and the time when clot firmness reaches $20 \mathrm{~mm}$. CT indicates the ability of thrombin generation and is prolonged by anticoagulants such as heparin. MCF represents maximum clot firmness which is defined by plasma fibrinogen level and platelet number and function

regression lines are relatively small, indicating that PT and APTT are not particularly sensitive to apixaban [1, 10]. The chromogenic anti-Xa assay is a functional test for anti-Xa activity, which has been reported to correlate well with plasma apixaban concentration $[10,11]$. The values of plasma anti-Xa activity after anesthesia induction and the end of surgery were $1.69 \mathrm{IU} / \mathrm{mL}$ and 1.12 $\mathrm{IU} / \mathrm{mL}$, respectively, in the present case (Table 1 ). It has been reported that the trough level of apixaban in patients with a dosage of $5 \mathrm{mg}$ twice daily is $1.0 \mathrm{IU} / \mathrm{mL}$ [12-14]. Hence, the antithrombotic property of apixaban was considered to be in the therapeutic range during the present surgery. It might be desirable to perform a chromogenic anti-Xa assay to estimate perioperative bleeding risk owing to apixaban; however, it is usually feasible only in a specialized laboratory and has a long turnaround time. We sent blood samples for anti-Xa assay to an outside institution and obtained results several days later.

We examined the potential of an RVV test performed with ClotPro for evaluation of the antithrombotic effect of apixaban because a point-of-care device with a short turnaround time is suitable for the perioperative period. The RVV test has been developed on the same principle as RVV time (RVVT), which is another functional test for the evaluation of anti-Xa activity; RVVT is an assay for the detection of lupus anticoagulants, but it is also affected by anti-Xa drugs, including apixaban, because RVV specifically cleaves factor $\mathrm{X}$ to $\mathrm{Xa}[3,4,15]$. As shown in Table 1, the results of the RVV test after anesthesia induction showed prolonged $\mathrm{CT}$, which also showed a proportional linear relation to anti-Xa activity in the therapeutic range of apixaban (Fig. 2). These results might indicate feasibility of the RVV test as a point-of-care assay for evaluation of the anticoagulant effect of apixaban, although an appropriate observational study is required to validate its clinical usefulness.

Because the end point for determination of $\mathrm{CT}$ is fibrin clot formation, similar to other viscoelastic tests, prolonged CT with an RVV test might not always reflect anti-Xa activity. Considering the coagulation cascade, it

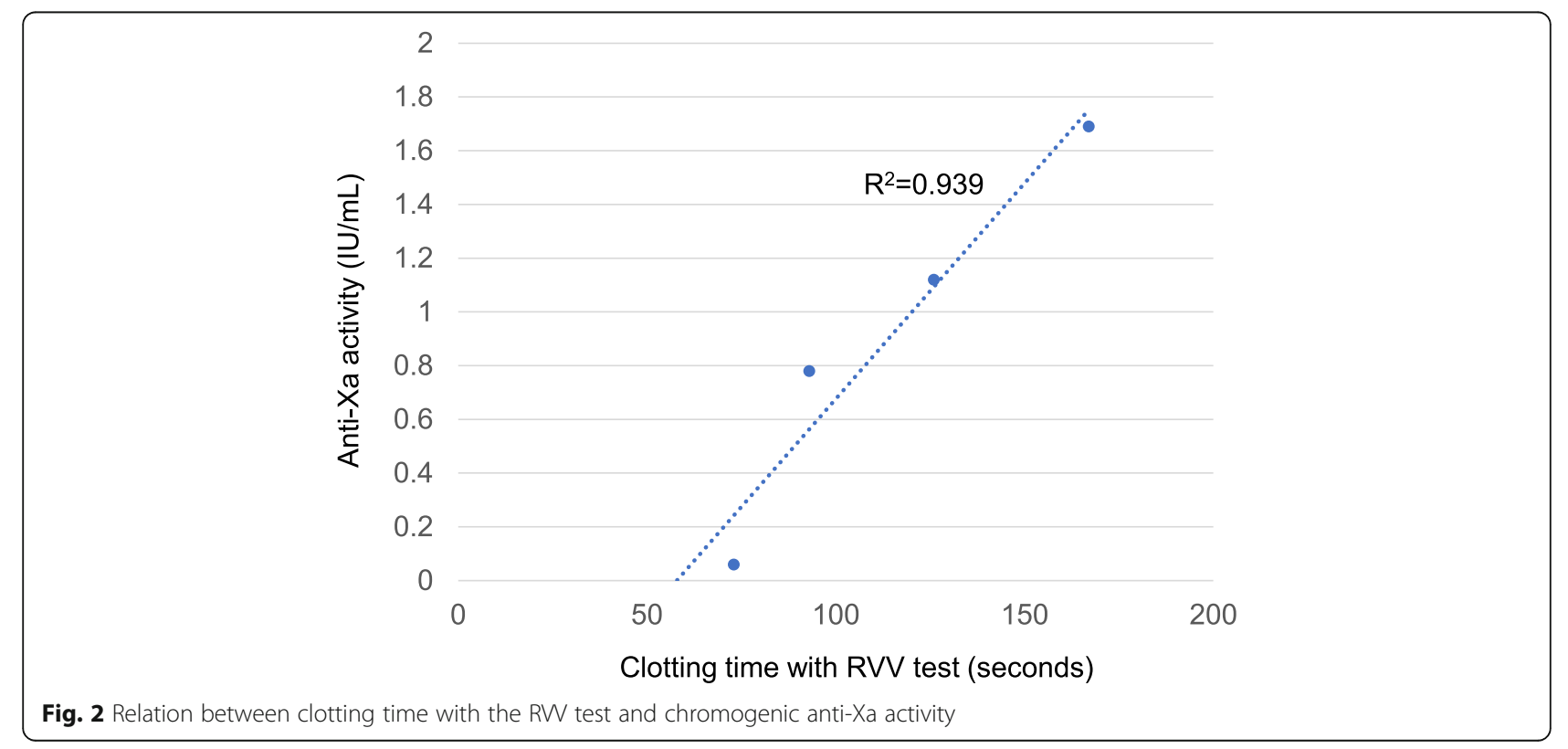


could be also affected by anti-thrombin drugs, such as argatroban or dabigatran, or by hypofibrinogenemia. Thus, we also estimated anti-thrombin activity with the ECA and examined the plasma fibrinogen level [5]. The results were consistently in the normal range during the perioperative period, confirming that the prolongation of CT in the RVV test reflected anti-Xa activity owing to apixaban. Thus, it is reasonable to perform the RVV test in combination with the ECA to specifically evaluate anti-Xa activity.

In conclusion, we described the evaluation of the residual anti-Xa effect of apixaban with an RVV test using ClotPro for a patient who had a minimal interruption period of apixaban perioperatively. Given that continuation of anti-Xa drugs is generally accompanied by increased risks of bleeding and transfusion, the point-ofcare evaluation of anti-Xa effect could help physicians prepare for massive bleeding. The short turnaround time for the RVV test using ClotPro might be useful for evaluation of the anti-Xa level induced by apixaban during the perioperative period. A future prospective study should elucidate the clinical usefulness of the RVV test.

\section{Abbreviations}

APTT: Activated partial thromboplastin time; CT: Clotting time;

POD: Postoperative day; PT: Prothrombin time; RW: Russell's viper venom; Xa: Activated factor $X$

\section{Acknowledgements}

Not applicable.

\section{Authors' contributions}

KS and NK wrote the manuscript; NK, YK, HS, and TT revised the manuscript; SU supervised this work; KS and SK prepared the manuscript files in consultation with NK, HS, TT, and SU. All authors reviewed and approved the final manuscript for submission.

\section{Funding}

The authors declare that they have no funding.

\section{Availability of data and materials}

Not applicable.

\section{Declarations}

Ethics approval and consent to participate

The Ethics Committee of Jikei University approved the publication of this case report.

\section{Consent for publication}

Written informed consent was obtained from the patient for publication of this case report.

\section{Competing interests}

The authors declare that they have no competing interests.

\section{Author details}

'Department of Anesthesiology, The Jikei University School of Medicine, 3-25-8 Nishishinbashi, Minato-ku, Tokyo 105-8461, Japan. ${ }^{2}$ Department of Orthopedics, The Jikei University School of Medicine, 3-25-8 Nishishinbashi, Minato-ku, Tokyo 105-8461, Japan.
Received: 1 April 2021 Revised: 23 April 2021

Accepted: 26 April 2021 Published online: 06 May 2021

\section{References}

1. Testa S, Legnani C, Tripodi A, Paoletti O, Pengo V, Abbate R, et al. Poor comparability of coagulation screening test with specific measurement in patients receiving direct oral anticoagulants: results from a multicenter/ multiplatform study. J Thromb Haemost. 2016;14(11):2194-201. https://doi. org/10.1111/jth.13486.

2. Dubois V, Dincq AS, Douxfils J, Ickx B, Samama CM, Dogné JM, et al. Perioperative management of patients on direct oral anticoagulants. Thromb J. 2017;15(1):14. https://doi.org/10.1186/s12959-017-0137-1.

3. Pratt J, Crispin P. Screening test for direct oral anticoagulants with the dilute Russell viper venom time. Eur J Haematol. 2018;100(6):567-74. https://doi. org/10.1111/ejh.13050

4. Marsh NA. Diagnostic uses of snake venom. Haemostasis. 2001;31(3-6):2117. https://doi.org/10.1159/000048065.

5. Fong AYY, Tiong LL, Tan SSN, Geruka D, Apil GG, Choo CW, et al. Effect of dabigatran on clotting time in the ClotPro ecarin clotting assay: a prospective, single-arm, open-label study. Clin Appl Thromb Hemost. 2020; 26:1076029620972473.

6. Raval AN, Cigarroa JE, Chung MK, Diaz-Sandoval LJ, Diercks D, Piccini JP, et al. Management of patients on non-vitamin $\mathrm{K}$ antagonist oral anticoagulants in the acute care and periprocedural setting: a scientific statement from the American Heart Association. Circulation. 2017;135(10): e604-33.

7. Steffel J, Verhamme P, Potpara TS, Albaladejo P, Antz M, Desteghe L, et al. The 2018 European Heart Rhythm Association Practical Guide on the use of non-vitamin $\mathrm{K}$ antagonist oral anticoagulants in patients with atrial fibrillation. Eur Heart J. 2018;39(16):1330-93. https://doi.org/10.1093/eurhea rtj/ehy136.

8. Frost C, Nepal S, Wang J, Schuster A, Byon W, Boyd RA, et al. Safety, pharmacokinetics and pharmacodynamics of multiple oral doses of apixaban, a factor Xa inhibitor, in healthy subjects. Br J Clin Pharmacol. 2013;76(5):776-86. https://doi.org/10.1111/bcp.12106.

9. Samuelson BT, Cuker A, Siegal DM, Crowther M, Garcia DA. Laboratory assessment of the anticoagulant activity of direct oral anticoagulants: a systematic review. Chest. 2017;151(1):127-38. https://doi.org/10.1016/j. chest.2016.08.1462.

10. Yamahira N, Frost C, Fukase H, Yu Z, Wang J, Pursley J, et al. Safety, tolerability, pharmacokinetics, and pharmacodynamics of multiple doses of apixaban in healthy Japanese male subjects. Int I Clin Pharmacol Ther. 2014; 52(7):564-73. https://doi.org/10.5414/CP201967.

11. Conway SE, Hwang AY, Ponte CD, Gums JG. Laboratory and clinical monitoring of direct acting oral anticoagulants: what clinicians need to know. Pharmacotherapy. 2017;37(2):236-48. https://doi.org/10.1002/phar.1884.

12. Byon W, Sweeney K, Frost C, Boyd RA. Population pharmacokinetics, pharmacodynamics, and exploratory exposure-response analyses of apixaban in subjects treated for venous thromboembolism. CPT Pharmacometrics Syst Pharmacol. 2017;6(5):340-9. https://doi.org/10.1002/ psp4.12184

13. Osanai H, Ajioka M, Masutomi T, Kuwayama T, Ishihama S, Sakamato Y, et al. Measurement of anti-factor Xa activity in patients on apixaban for nonvalvular atrial fibrillation. Circ J. 2015;79(12):2584-90. https://doi.org/10.1253/ circj.CJ-15-0470.

14. Ikeda K, Tachibana H. Clinical implication of monitoring rivaroxaban and apixaban by using anti-factor Xa assay in patients with non-valvular atrial fibrillation. J Arrhythm. 2016;32(1):42-50. https://doi.org/10.1016/j.joa.2015. 08.001 .

15. Favaloro EJ. The Russell viper venom time (RWT) test for investigation of lupus anticoagulant (LA). Am J Hematol. 2019;94(11):1290-6. https://doi. org/10.1002/ajh.25606.

\section{Publisher's Note}

Springer Nature remains neutral with regard to jurisdictional claims in published maps and institutional affiliations. 